\title{
Evaluation des teneurs des métaux lourds dans le sang des sujets de la zone de traitement des phosphates au Sud du Togo
}

\author{
Adoté Agbéko ADUAYI-AKUE ${ }^{1,2^{*}}$, Kissao GNANDI ${ }^{1,2}$, Amivi TETE-BENISSAN ${ }^{3}$, \\ Mlatovi DEGBE ${ }^{3}$, Gnon TANOUAYI ${ }^{1,2}$ et Messanvi GBEASSOR ${ }^{3}$ \\ ${ }^{I}$ Laboratoire de Gestion, Traitement et Valorisation des Déchets (GTVD), Faculté des Sciences, \\ Université de Lomé, Togo. \\ ${ }^{2}$ Laboratoire de Géosciences, Faculté des Sciences, Université de Lomé, Togo. \\ ${ }^{3}$ Laboratoire de Physiologie Animale et Pharmacologie, Faculté des Sciences, Université de Lomé, Togo. \\ *Auteur correspondant, E-mail: alexisaduayi@yahoo.fr
}

\section{RESUME}

Les déchets du traitement des phosphates au Togo renferment des métaux lourds à l'origine de la contamination des sols, l'air, l'eau, la faune et la flore. Ce qui constitue un danger pour la santé des populations exposées. La présente étude a pour but d'évaluer les taux sanguins des métaux lourds chez les sujets exposés. Ainsi, les teneurs en cadmium, plomb, cuivre et nickel du sang ont été déterminées au spectrophotomètre d'absorption atomique chez 260 sujets dont 173 employés, 60 riverains de l'usine et 27 sujets témoins. Les résultats montrent que les valeurs moyennes des métaux lourds sont significativement plus élevées $(\mathrm{p}<0,001)$ chez les travailleurs et les riverains de l'usine. La prévalence de la bioconcentration chez les sujets exposés varie en fonction du degré de contamination par les poussières de phosphate. Elle serait liée soit à la consommation d'aliments contaminés, soit à la durée de l'exposition aux métaux lourds. Ces résultats confirment une contamination importante et des risques sanitaires graves chez les sujets étudiés. Ce qui devrait permettre la mise en place dans l'usine de programmes efficaces et des mesures adéquates pour une meilleure protection des employés et une réduction sensible de la pollution des localités riveraines.

(C) 2015 International Formulae Group. All rights reserved.

Mots clés : Phosphates, métaux lourds, bioconcentration, Sud Togo.

\section{Evaluation of heavy metal concentrations in the blood of subjects in the area of processing of phosphates in southern Togo}

\begin{abstract}
The waste from the processing of phosphates in Togo contains heavy metals causing the contamination of soil, air, water, flora and fauna. This constitutes a danger to the health of exposed populations. This study aimed at evaluating blood levels of heavy metals in exposed subjects. Thus, the blood levels of cadmium, lead, copper and nickel were determined using atomic absorption spectrophotometer in 260 subjects, including 173 employees, 60 residents around the factory and 27 control subjects. The results show that the average values of heavy metals were significantly higher $(\mathrm{p}<0.001)$ among workers and residents around the factory. The variation of prevalence of bioconcentration in exposed subjects depends on the degree of contamination by phosphate dust. It would be linked to the consumption of contaminated food, or the duration of exposure to
\end{abstract}


heavy metals. These results confirmed significant contamination and serious health risks in the studied subjects. This should allow the introduction, in the plant, of effective programs and appropriate measures for better protection of employees and a significant reduction in pollution of areas surrounding the factory.

(C) 2015 International Formulae Group. All rights reserved.

Keywords: Phosphates, heavy metals, bioconcentration, South Togo.

\section{INTRODUCTION}

Le continent africain est un producteur important et incontournable d'un grand nombre de matières premières minières. Ceci donne l'opportunité aux pays africains d'augmenter les recettes d'exportation et de lutter contre la pauvreté. Le Togo, petit pays de l'Afrique de l'Ouest dont le sous-sol ne regorge pas d'importants gisements miniers, tire de l'exploitation du minerai de phosphate, des recettes non négligeables qui contribuent pour une part importante à son développement économique. Cependant, les activités d'exploitation et de traitement de ce minerai sont à l'origine de la contamination des sols, l'air, l'eau, la faune, la flore et ont un impact négatif sur l'environnement (DP/MERF, 2010). En effet, des études antérieures ont montré la présence de métaux lourds toxiques dans le minerai de phosphates du Sud Togo, ce qui constitue un danger pour l'environnement et la santé humaine. Les organismes vivants s'intoxiquent par l'alimentation, la respiration et l'absorption cutanée (Gnandi et Tobschall, 2003; Tchangbedji et al., 2003 ; Verloo, 2003; Aduayi-Akue et Gnandi, 2014), ceci entraîne des perturbations aigues ou chroniques chez l'homme (Testud, 2005 ; Farombi et al., 2007). Par conséquent, la pollution du milieu naturel constitue un problème majeur de santé publique qui préoccupe les autorités dans la plupart des pays (Boudene, 2001 ; Miquel, 2001 ; Alinor, 2005). Ainsi, plusieurs études ont aussi mis en évidence les variations des concentrations de métaux lourds dans le sang de sujets contaminés (Durand et al., 2008; Gallagher et al., 2008 ; Fadrowski et al., 2010 ; Spector et al., 2011).

$\mathrm{Au}$ Togo, de nombreux travaux ont mis en évidence dans la zone d'exploitation et de traitement des phosphates, la contamination par les métaux lourds des sols, des eaux de surface et souterraines, de la flore et de la faune aquatique (Gnandi et Tobschall, 2002; Gnandi et al., 2006a; Mélila et al., 2012a; Aduayi-Akue et Gnandi, 2014; Tanouayi et al., 2015). D'autres études ont révélé que cette contamination, entraîne des perturbations physiologiques et l'augmentation du stress oxydatif chez les populations riveraines et la faune aquatique (Gnandi et al., 2006b; Mélila et al., 2012b). Malgré tous ces travaux, il manque encore des données sur les valeurs des teneurs des métaux lourds dans le sang des sujets exposés.

Le présent travail a pour but d'évaluer sur la côte atlantique du Togo, dans un échantillon de population de la zone contaminée, les taux sanguins de cadmium, plomb, cuivre, nickel et de déterminer les facteurs de variation de leurs concentrations respectives chez les sujets plus ou moins exposés aux poussières de phosphate. Les données de cette étude permettront une meilleure appréciation des problèmes de sécurité sanitaire liés à l'exploitation minière et la mise en place des programmes adéquats pour une prise en charge efficace des populations.

\section{MATERIEL ET METHODES La zone d'étude}

Elle est représentée sur la Figure 1 et située sur la côte atlantique au Sud-Est du Togo. Le climat de la région littorale est de type subéquatorial avec deux saisons de pluies et deux saisons sèches sous la dépendance de l'harmattan du Nord-Est, sec et chaud, soufflant de décembre à janvier et la mousson du Sud-Ouest, froid et humide, soufflant de juin à septembre et qui apporte la pluie. Dans la zone d'exploitation des phosphates, les moyennes des températures varient entre $25{ }^{\circ} \mathrm{C}$ et $29{ }^{\circ} \mathrm{C}$. L'humidité moyenne dépasse $75 \%$, l'évapotranspiration réelle est de $2013 \mathrm{~mm} / \mathrm{an}$ en moyenne. Le relief de la zone d'étude présente une altitude 
parfois inférieure au niveau de la mer et ne dépasse pas $15 \mathrm{~m}$. L'hydrographie est constituée de plusieurs lacs et cours d'eau exploités par les populations pour les besoins de la pêche. Le littoral du Togo présente une diversité floristique tant pour les formations sur terre ferme que pour les écosystèmes marins et aquatiques. La végétation de la plaine côtière revêt un caractère mosaïque. Les cultures maraîchères, de céréales et de tubercules constituent l'essentiel des activités agricoles.

\section{Matériel}

\section{La population d'étude}

Elle est constituée de 260 sujets adultes des deux sexes non apparentés, apparemment sains, issus de diverses couches socioprofessionnelles, âgés de 22 à 60 ans, avertis, renseignés, sensibilisés sur l'étude et ayant tous donné leur consentement après l'autorisation du Comité de Bioéthique (N753/2014). Ils sont répartis en plusieurs groupes: groupe I formé de 173 employés travaillant sur le site de l'usine (chaîne de fabrication: Fab, atelier de maintenance : Mai, administration : Adm), groupe II : 60 sujets vivant dans les villages riverains (Aglomé : Agl, Goumoukopé : Gou), groupe III : 27 sujets témoins habitant une localité (Gbodjomé : Gbo) non exposée à la contamination située en amont, à $11 \mathrm{~km}$ de l'usine de traitement.

Les sujets sélectionnés travaillent ou vivent dans la zone d'étude depuis au moins cinq ans. La durée d'exposition correspond aux années de service passées dans l'usine pour le groupe I et le temps de séjour des riverains (groupe II) dans les villages concernés. Une enquête nutritionnelle préalable a été réalisée auprès des différents groupes. Sont exclus de l'étude tous les consommateurs réguliers d'alcool et les fumeurs.

\section{Matériel biologique}

Dix (10) ml de sang ont été prélevés par ponction veineuse au pli du coude sur des sujets assis et à jeun depuis au moins 12 heures et recueillis dans un tube garanti sans métal lourd contenant un anticoagulant (EDTA). Les échantillons ont été conservés à $4{ }^{\circ} \mathrm{C}$ dans une glacière isotherme contenant de la glace jusqu'au laboratoire pour les analyses. A la suite de la prise de sang, tous les sujets ont fait l'objet d'un interrogatoire nutritionnel sur tous les repas pris au cours des 48 heures précédant le prélèvement.

\section{Dosage des métaux lourds}

Il est réalisé selon la méthode de Labat (2010). Dans un bécher en téflon, $4 \mathrm{ml}$ de $\mathrm{HNO}_{3}$ à $65 \%$ (Labosi Oulshy-LechâteauFrance) sont additionnés à $5 \mathrm{ml}$ d'échantillon de sang. La minéralisation est effectuée dans un «bain de sable» à $150{ }^{\circ} \mathrm{C}$ pendant au moins 2 heures jusqu'à l'évaporation du surnageant. Ensuite pour faciliter la dissolution du culot, $2 \mathrm{ml}$ de $\mathrm{HNO}_{3}$ à $65 \%$ et $2 \mathrm{ml}$ de $\mathrm{H}_{2} \mathrm{O}$ distillée sont ajoutés au culot qui est de nouveau chauffé pendant $20 \mathrm{mn}$. Le mélange refroidi à température ambiante pendant $15 \mathrm{mn}$ est ramené à $5 \mathrm{ml}$ avec $\mathrm{H}_{2} \mathrm{O}$ distillée. Après filtration sur papier (MN 640 dd, Machery-Nagel+Co, Germany), les métaux lourds (cadmium, plomb, cuivre, nickel) sont dosés au Spectrophotomètre d'Absorption Atomique Solaar S2 (Thermo Electron Corporation ; Orion, England). Des solutions étalons (Merck KGaA ; Durmstalt, Allemagne) des différents métaux ont servi de standards. Seuils de détection des métaux pour l'appareil : plomb : 0,01 $\mathrm{mg} / \mathrm{l}$; cadmium : 0,0005 mg/l ; cuivre : 0,01 mg/l ; nickel : 0,0004 mg/l.

\section{Traitement des données}

L'analyse statistique a été réalisée à l'aide du logiciel GraphPad Prism 6.0. Les résultats quantitatifs sont exprimés par la moyenne et l'écart-type. Les écarts entre les différents groupes ont été comparés à l'aide du test d'ANOVA suivi du post-test de Tuckey pour la comparaison des différents groupes entre eux, le seuil de signification retenu est de $5 \%$.

Le facteur de bioaccumulation (FB) a été calculé selon la formule suivante :

$\mathrm{FB}=$ Concentration sanguine moyenne du métal / Valeur de la norme du métal.

FB permet de préciser le degré de bioconcentration. On considère qu'il y a risque sanitaire lorsque la valeur de FB $>1$. 
A. A. ADUAYI-AKUE et al. / Int. J. Biol. Chem. Sci. 9(4): 1972-1982, 2015

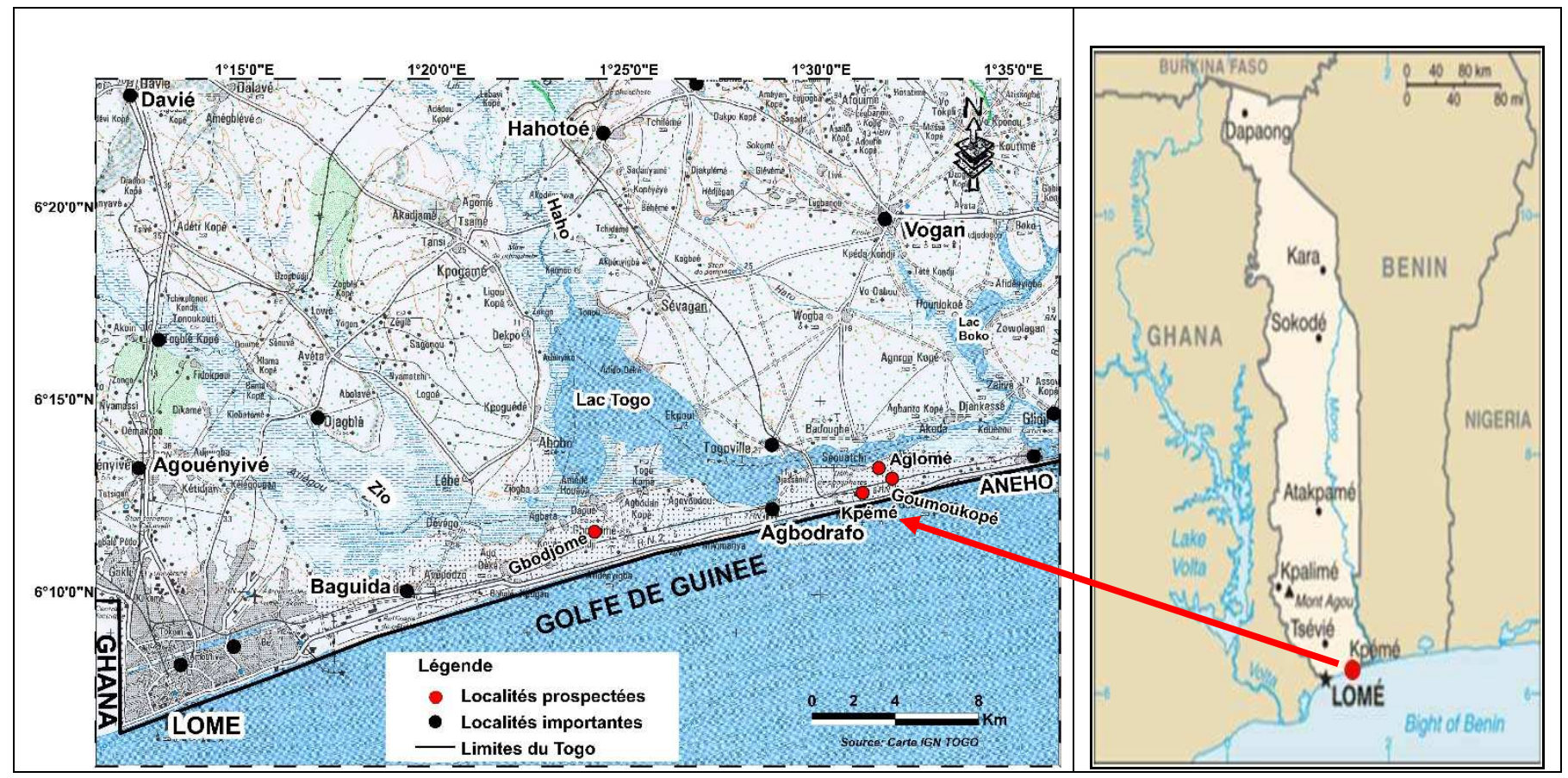

Figure 1 : Carte montrant la zone d'étude avec les différents points d'échantillonnage. 


\section{RESULTATS}

Habitudes alimentaires de la population d'étude

Les enquêtes et interrogatoires nutritionnels réalisés chez tous les sujets montrent que dans la zone d'étude, les apports glucidiques (maïs, manioc, légumes, fruits...) et protéiques (poissons, crustacés, moules et volailles) proviennent essentiellement des cultures sur sols pollués et de la faune contaminée. Les besoins en lipides sont essentiellement couverts par les huiles de palme et de coco produites sur place.

\section{Détermination des teneurs des métaux lourds dans le sang}

Les Tableaux ( 1 et 2 ), la Figure 2 indiquent les teneurs moyennes et les valeurs extrêmes des métaux lourds dans le sang des sujets de l'étude. Le Tableau 3 montre les valeurs des facteurs de bioaccumulation (FB) chez tous les sujets.

Les résultats révèlent dans l'ordre décroissant les valeurs moyennes des teneurs $\mathrm{du}$ cadmium $(\mathrm{Cd})$, plomb $(\mathrm{Pb})$, nickel $(\mathrm{Ni})$ et cuivre $(\mathrm{Cu})$ dans les deux groupes (usine et riverains) $\mathrm{Pb}>\mathrm{Cu}>\mathrm{Cd}>\mathrm{Ni}$ qui sont significativement $(\mathrm{p}<0,001)$ plus élevées que celles des témoins; avec des facteurs de bioaccumulation $(\mathrm{FB})>$ à 1 , sauf pour le $\mathrm{Cu}$. $\mathrm{Le} \mathrm{Cd}$ a le $\mathrm{FB}$ le plus élevé $(\mathrm{Cd}>\mathrm{Pb}>\mathrm{Ni}>$ $\mathrm{Cu}$ ). Cependant chez les sujets témoins (Gbo), les valeurs observées sont plus faibles que les seuils supérieurs recommandés.

Les teneurs des métaux lourds et les facteurs de bioaccumulation se présentent dans l'ordre décroissant suivant le lieu d'exposition: Aglomé > chaîne de fabrication $>$ maintenance > Goumoukopé > administration > Gbodjomé. Pour le nickel, les sujets de l'administration (Adm) de l'usine ont des teneurs inférieures au seuil recommandé. Il en est de même pour le cuivre chez tous les sujets.

Chez les sujets du groupe I (usine) : les sujets de la chaine de fabrication présentent des valeurs significativement plus élevées : Ni et $\mathrm{Cd}(\mathrm{p}<0,001)$; $\mathrm{Cu}$ et $\mathrm{Pb}(\mathrm{p}<0,01)$ que celles des sujets de la maintenance et de l'administration (Fab > Mai > Adm). Des différences significatives sont observées $(\mathrm{p}<$ $0,01)$ pour $\mathrm{Ni}$ et $\mathrm{Cd},(\mathrm{p}<0,05)$ pour le $\mathrm{Pb}$ entre les sujets de la maintenance et de l'administration.

Chez les sujets du groupe II (riverains de l'usine) : les teneurs du $\mathrm{Cd}$ et $\mathrm{du} \mathrm{Ni}$ sont significativement plus élevées $(p<0,001)$ à Aglomé (Agl) par rapport à Goumoukopé (Gou).

\section{Variation des teneurs en fonction de la durée d'exposition aux métaux lourds}

La Figure 3 montre les variations des teneurs en fonction de la durée d'exposition aux métaux lourds dont les valeurs moyennes dans le sang et les facteurs de bioaccumulation augmentent avec le nombre d'années d'exposition.

- Pour le Cd, les différences significatives ( $\mathrm{p}<$ $0,001)$ sont observées dans les groupes I et II pour les durées < à 21 ans.

- En ce qui concerne le $\mathrm{Pb}$, les différences observées dans les groupes I et II sont significatives $(\mathrm{p}<0,05)$ sauf pour les sujets de l'administration $(\mathrm{p}>0,05)$ pour les durées d'exposition $>21$ ans

- Dans les groupes I et II, pour le nickel, des différences significatives $(\mathrm{p}<0,05)$ sont observées sauf à Goumoukopé (Gou).

- Concernant le cuivre, les différences sont significatives $(p<0,001)$ dans les groupes I et II. 
Tableau 1: Teneurs en métaux lourds dans le sang des différents groupes de sujets (moyenne \pm écart type).

\begin{tabular}{|c|c|c|c|c|}
\hline Groupes de sujets & $\mathrm{Cd}(\mu \mathrm{g} / \mathrm{l})$ & $\mathrm{Pb}(\mu \mathrm{g} / \mathrm{l})$ & $\mathrm{Ni}(\mu \mathrm{g} / \mathrm{l})$ & $\mathrm{Cu}(\mu \mathrm{g} / \mathrm{l})$ \\
\hline Fab $(n=87)$ & $19,48 \pm 7,04 * * * a$ & $334,92 \pm 81,70 * * a$ & $18,06 \pm 4,60 * * * a$ & $302,81 \pm 122 * * a$ \\
\hline Mai $(n=56)$ & $14,79 \pm 5,80 * * a$ & $330,10 \pm 81,61 * a$ & $11,95 \pm 2,02 * * \mathrm{a}$ & $276,70 \pm 105 \mathrm{Ns} \mathrm{a}$ \\
\hline $\operatorname{Adm}(n=30)$ & $9,58 \pm 3,68$ & $276,11 \pm 99,53$ & $8,60 \pm 1,12$ & $213,70 \pm 92,81$ \\
\hline $\operatorname{Agl}(n=36)$ & $24,02 \pm 7,06 * * * b$ & $361,50 \pm 83,10 \mathrm{Ns} \mathrm{b}$ & $23,14 \pm 5,78 * * * b$ & $342,31 \pm 132 \mathrm{Ns} \mathrm{b}$ \\
\hline Gou $(n=24)$ & $14,25 \pm 5,49$ & $315,23 \pm 70,32$ & $11,11 \pm 4,05$ & $304,22 \pm 116$ \\
\hline Gbo $(n=27)$ & $2,04 \pm 0,55$ & $105,80 \pm 40$ & $4,28 \pm 1,26$ & $180 \pm 80,22$ \\
\hline Valeurs seuils & 5 & 200 & 10 & 700 \\
\hline Réf. & OMS (1980) & OMS (1980) & Biotox (2014) & Biomnis (2012) \\
\hline
\end{tabular}

Tableau 2 : Valeurs extrêmes des teneurs en métaux dans le sang des sujets de l'étude.

\begin{tabular}{lcccc}
\hline Groupes de sujets & $\mathbf{C d}(\boldsymbol{\mu g} / \mathbf{l})$ & $\mathbf{P b}(\boldsymbol{\mu g} / \mathbf{l})$ & $\mathbf{N i}(\boldsymbol{\mu g} / \mathbf{l})$ & $\mathbf{C u}(\boldsymbol{\mu g} / \mathbf{l})$ \\
\hline Fab $(\mathrm{n}=87)$ & $8,07-28,96$ & $191,96-491,32$ & $11,51-26,88$ & $148,76-531,42$ \\
Mai $(\mathrm{n}=56)$ & $0,18-22,61$ & $189,09-486$ & $7,18-14,87$ & $120,53-489,23$ \\
Adm $(\mathrm{n}=30)$ & $2,47-16,91$ & $118,77-385,08$ & $7,05-10,82$ & $94,30-348,21$ \\
\hline Agl $(\mathrm{n}=36)$ & $11,44-33,37$ & $208,17-475,08$ & $13,1-32,11$ & $146,04-543,74$ \\
Gou (n= 24) & $4,78-22,50$ & $184,20-399,27$ & $2,29-19,90$ & $147,48-471,22$ \\
\hline Gbo (n= 27) & $1,05-2,90$ & $50,81-156,31$ & $2,48-7,08$ & $79,39-293,27$ \\
\hline Valeurs seuils & 5 & 200 & 10 & 700 \\
Réf. & OMS (1980) & OMS (1980) & Biotox (2014) & Biomnis $(2012)$ \\
\hline n= effectif; Cd= Cadmium ; Pb= Plomb; Ni= Nickel; Cu= Cuivre ; Fab= Fabricant ; Mai= Maintenancier ; Adm= \\
Administratif; Agl= Aglomé ; Gou= Goumoukopé ; Gbo= Gbodjomé (témoins).
\end{tabular}

Tableau 3 : Facteurs de bioaccumulation des métaux lourds.

\begin{tabular}{lcccc}
\hline Groupes de sujets & $\mathbf{C d}$ & $\mathbf{P b}$ & $\mathbf{N i}$ & $\mathbf{C u}$ \\
\hline Fab $(\mathrm{n}=87)$ & $3,89 \pm 1,4$ & $1,67 \pm 0,4$ & $1,8 \pm 0,46$ & $0,43 \pm 0,17$ \\
Mai $(\mathrm{n}=56)$ & $2,95 \pm 1,17$ & $1,65 \pm 0,4$ & $1,19 \pm 0,2$ & $0,39 \pm 0,15$ \\
Adm $(\mathrm{n}=30)$ & $1,91 \pm 0,73$ & $1,38 \pm 0,49$ & $0,86 \pm 0,11$ & $0,3 \pm 0,13$ \\
\hline Agl $(\mathrm{n}=36)$ & $4,8 \pm 1,41$ & $1,8 \pm 0,41$ & $2,31 \pm 0,57$ & $0,48 \pm 0,18$ \\
Gou $(\mathrm{n}=24)$ & $2,85 \pm 1,09$ & $1,57 \pm 0,35$ & $1,11 \pm 0,4$ & $0,43 \pm 0,16$ \\
Gbo $(\mathrm{n}=27)$ & $0,40 \pm 0,11$ & $0,52 \pm 0,2$ & $0,42 \pm 0,12$ & $0,25 \pm 0,11$ \\
\hline
\end{tabular}

$\mathrm{n}=$ effectif ; $\mathrm{Cd}=$ Cadmium ; $\mathrm{Pb}=$ Plomb $\mathrm{Ni}=\mathrm{Nickel} ; \mathrm{Cu}=$ Cuivre ; Fab= Fabricant ; Mai= Maintenancier ; Adm= Administratif ; Agl= Aglomé ; Gou= Goumoukopé ; Gbo= Gbodjomé (population témoin). 

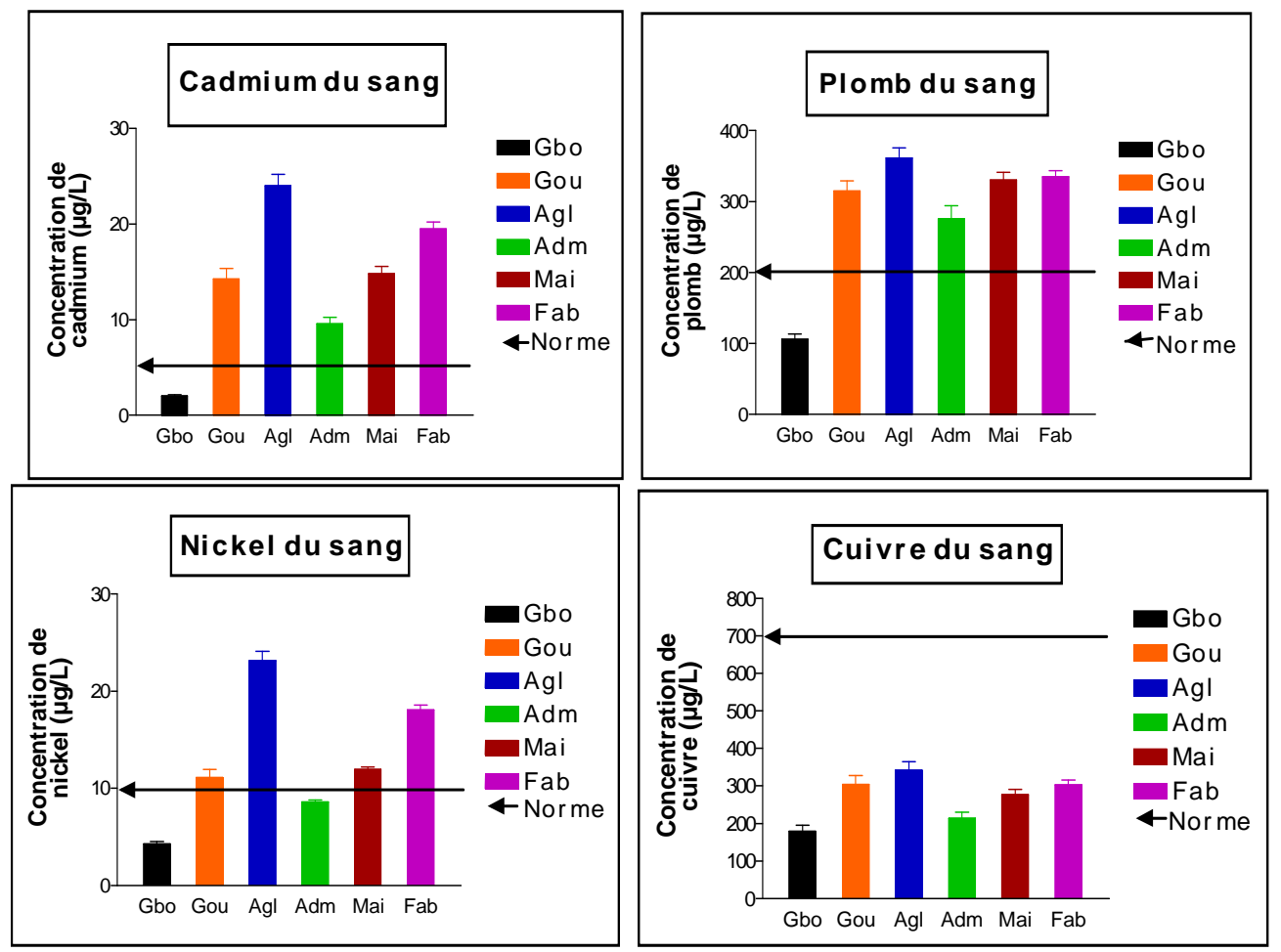

Figure 2 : Histogrammes des teneurs moyennes des métaux lourds des différents groupes de sujets. $\mathrm{Gbo}=$ Gbodjomé (population témoin); Gou= Goumoukopé ; Agl= Aglomé ; Adm= Administratif ; Mai= Maintenancier ; $\mathrm{Fab}=$ Fabricant.
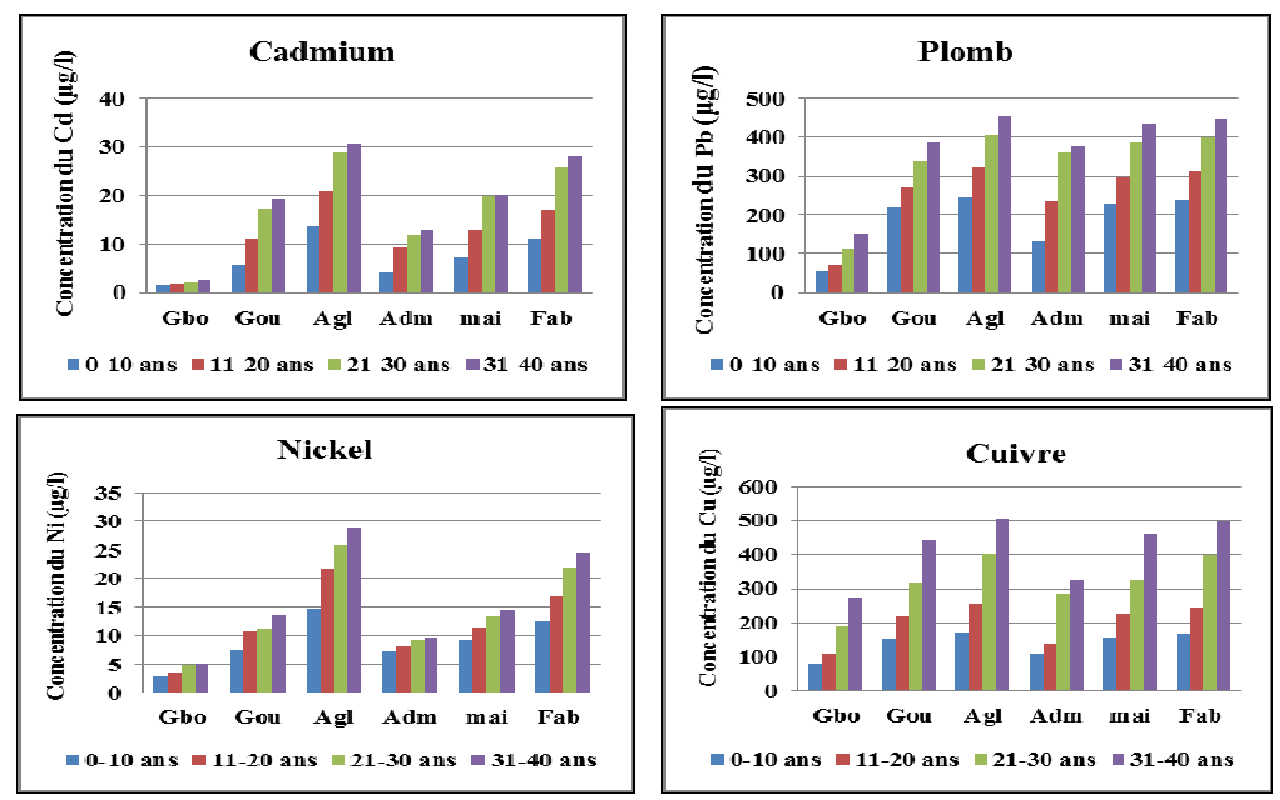

Figure 3 : Histogrammes des teneurs moyennes de métaux lourds suivant la durée d'exposition. Gbo= Gbodjomé (population témoin); Gou= Goumoukopé ; Agl= Aglomé ; Adm= Administratif ; Mai= Maintenancier ; $\mathrm{Fab}=$ Fabricant. 


\section{DISCUSSION}

Les teneurs en métaux lourds dans le sang des sujets employés de l'usine et des riverains ont été déterminées en fonction du temps d'exposition aux poussières de phosphates et de la qualité des aliments consommés. En effet, les résultats des enquêtes nutritionnelles réalisées auprès de la population d'étude montrent qu'environ 55\% à $65 \%$ des sujets du groupe I (employés de l'usine) dont les revenus sont élevés consomment des produits alimentaires (céréales, tubercules, fruits, légumes, poissons, crustacés..) en provenance des zones éloignées du site de l'usine. De ce fait, ils se contaminent moins par l'alimentation. En revanche, les sujets du groupe II riverains de l'usine aux revenus faibles consomment essentiellement les produits agricoles, de la pêche et de l'élevage provenant de la zone contaminée par les poussières de phosphates. Ils sont susceptibles de se contaminer aussi par l'alimentation. En effet, des études antérieures ont révélé que l'eau et les produits alimentaires (poissons, crustacés, céréales, fruits et légumes) provenant des localités riveraines sont contaminés par les métaux lourds des phosphates (Gnandi et al., 2006a ; Mélila et al., 2012a ; Aduayi-Akue et Gnandi, 2014 ; Tanouayi, 2015).

L'analyse des résultats du dosage des métaux lourds dans le sang des sujets de l'étude révèlent que le cadmium, le plomb, le nickel et le cuivre se retrouvent dans le sang des sujets de la population d'étude. Les teneurs en métaux lourds dans le sang des sujets témoins (Gbo) sont inférieures aux seuils supérieurs recommandés par l'OMS (1980), Biomis (2012) et Biotox (2014). Ces données se rapprochent de celles des travaux antérieurs réalisés sur les sols et le maïs cultivés dans la zone d'étude (Aduayi-Akue et Gnandi, 2014). Les valeurs moyennes observées chez les sujets de la zone contaminée sont significativement plus élevées $(\mathrm{p}<0,001)$ que celles des sujets témoins (Gbo): (Aglomé > fabricant > maintenancier > Goumoukopé > administrateur > Gbodjomé). Ceci suggère que les sujets de Gbodjomé ne sont presque pas contaminés. En effet, le village de ces derniers est situé vers l'Ouest à $11 \mathrm{~km}$ environ, en amont de l'usine, éloigné aussi des localités riveraines et est supposé ne pas recevoir de poussières de phosphates. De plus, il n'est pas non plus directement touché par le rejet des déchets de l'usine, ce qui explique son choix comme zone de référence (témoin). Ceci confirme les travaux antérieurs réalisés sur les poissons, les sols et le maïs de la zone d'étude (Mélila et al., 2012a ; Aduayi-Akue et Gnandi, 2014).

Les sujets du groupe I, employés de l'usine (fabricants, maintenanciers, administratifs) habitent à l'ouest à $1 \mathrm{~km}$ en amont du site dans une zone moins exposée à la pollution. Les valeurs significativement différentes observées chez ces derniers s'expliqueraient par le fait qu'ils sont exposés à différents degrés aux poussières de phosphates sur leur lieu de travail. En effet, tous les sujets du groupe I respirent l'air pollué du site. Quant aux employés de la chaîne de fabrication, ils sont plus en contact direct avec le minerai de phosphate. Ceux de la maintenance sont en plus exposés aux poussières retrouvées sur les machines et outils. Ceci confirme les teneurs en métaux lourds et le facteur de bioaccumulation significativement plus élevés observées chez les fabricants $(p<0,001)$ et les maintenanciers $(\mathrm{p}<0,01)$ par rapport aux administratifs. Bien que ces derniers travaillent dans l'usine, leurs valeurs plus faibles en métaux lourds s'expliqueraient par le fait qu'ils soient moins exposés (protection par les murs) par rapport aux autres employés.

Les sujets du groupe II (riverains de l'usine) habitent des villages situés dans un rayon de $3 \mathrm{~km}$ par rapport au site de traitement. Aglomé au Nord-Est, dans la direction du vent, est plus exposé aux poussières de phosphates. Goumoukopé à l'Est, reçoit moins de poussières par rapport à Aglomé. Cette localisation géographique des deux villages explique les valeurs significativement plus élevées $(p<0,001)$ des teneurs en métaux chez les sujets d'Aglomé 
qui respirent plus de poussières de phosphates et consomment des aliments plus contaminés en métaux lourds (Aduayi-Akue et Gnandi, 2014). En effet, des études antérieures ont montré que la contamination par voie respiratoire des éléments métalliques dans une atmosphère polluée peut être à l'origine d'une importante bioconcentration (Miquel, 2001 ; CCE, 2001). Les résultats de la présente étude confirment ceux des travaux effectués par El Abidi et al. (2000) qui ont trouvé que les populations riveraines d'une unité de fonderie de plomb sont plus contaminées que les ouvriers de l'usine.

Dans les trois groupes de sujets étudiés, les teneurs moyennes en métaux lourds du sang dépassent les valeurs des seuils sauf pour le $\mathrm{Cu}$. De plus, le cadmium est le métal le plus bioaccumulé, suivi du plomb $(\mathrm{Cd}>\mathrm{Pb}>\mathrm{Ni}>$ $\mathrm{Cu}$ ). Cependant, les résultats des travaux de Mélila et al. (2012b) sur les populations riveraines de notre zone d'étude avec un effectif plus réduit montrent des valeurs plus faibles et plus élevées respectivement pour le $\mathrm{Cd}$ et le $\mathrm{Pb}$. D'autres études réalisées au Maroc sur des populations riveraines d'une usine de phosphate dont le taux de Cd est 2,5 fois inférieur à celui du Togo $(55 \mathrm{ppm}$ vs 22 ppm) montrent que la contamination aussi en Cd est négligeable chez ces sujets $(1,1 \pm 0,7$ $\mu \mathrm{g} / \mathrm{l})$ (Khassouani et al., 2000). Bien que le $\mathrm{Pb}$ ait le plus faible facteur de pollution des sols et des cultures (maïs) dans la zone d'étude, son facteur de bioaccumulation est supérieur à ceux du Ni et du $\mathrm{Cu}$. Des études réalisées dans la zone révèlent que des quantités importantes de fumées et de gaz rejetées par l'usine renfermeraient des particules de $\mathrm{Pb}$ qui sont inhalés par tous sujets de la population d'étude (NSE/ONUDI, 2007).

\section{Conclusion}

Les résultats de cette étude confirment la contamination des employés et les riverains de l'usine de traitement des phosphates du Sud Togo. La bioconcentration de métaux lourds chez les sujets varie en fonction du poste occupé chez les employés de l'usine, de la localisation géographique chez les riverains et de la durée d'exposition de même que la qualité des aliments consommés pour tous les sujets. Le dosage des métaux lourds dans le sang montre que les teneurs en $\mathrm{Cd}, \mathrm{Pb}$ et $\mathrm{Ni}$ dépassent les valeurs seuils recommandées.

La bioaccumulation des métaux lourds entraînerait de graves risques sanitaires chez les populations. Des études complémentaires (environnementales, épidémiologiques et cliniques) permettront de mieux préciser les impacts environnementaux et les conséquences biologiques sur la santé des sujets pour une meilleure prise en charge sanitaire des populations exposées.

\section{Conflit d'intérêt}

Les auteurs déclarent qu'il n'y a aucun conflit d'intérêts pour la publication de l'article.

\section{Contributions des auteurs}

AAA-A, AT-B, MD et MG ont réalisé les enquêtes sociodémographiques et nutritionnelles, le prélèvement de sang humain, la conservation des échantillons de sang pour les analyses et la rédaction de l'article; AAA-A, KG et G T ont réalisé le dosage des métaux lourds dans le sang humain et la rédaction de l'article.

\section{REFERENCES}

Aduayi-Akue AA, Gnandi K. 2014. Evaluation de la pollution par les métaux lourds des sols et de la variété locale du maïs Zea mays dans la zone de traitement des phosphates de Kpémé (Sud du Togo). Int. J. Biol. Chem. Sci., 8(5): 2347-2355. DOI : http://dx.doi.org/10.4314/ijbcs.v8i5.37

Alinor IJ. 2005. Assessment of elemental contaminants in water and fish samples from Aba River. Environ Monit Assess., 102: $15-25$.

Biomnis 2012. Analyses Médicales Spécialisées. Précis de Biopathologie: Paris. 
Biotox 2014. Guide Biotoxicologique pour les Médecins du Travail : Canada. www.inrs.fr, [Consulté le 12/11/14].

Boudene C. 2001. Dossier scientifique sur la toxicité des métaux lourds toxiques. Science et vie, p. 24.

CCE (Commission des Communautés Européennes). 2001. Journal officiel des communautés européennes, règlement (CE) $\mathrm{N}^{\circ} 466 / 2001$ de la commission du 8 mars portant fixation de teneurs maximales pour certains contaminants dans les denrées alimentaires, p. 13.

DP/MERF (Direction de la Planification, Ministère de l'Environnement et des Ressources Forestières). 2010. Rapport national du Togo pour la dix huitième Session de la Commission de Développement Durable des Nations Unies (CDD-18), p. 44.

Durand C, Sauthier N, Schwoebel V. 2008. Evaluation de l'exposition à des sols pollués au plomb, au cadmium et à l'arsenic en Aveyron. Etude Cassiopée. Institut de veille sanitaire : Saint-Maurice. p. 307. http://www.invs.sante.fr, [consulté le 18/9/ 2014].

El Abidi A, Idrissi L, Taleb H, Azizi A, Mameli O, Melis P. 2000. The impact of lead pollution on the environment of Rabat-Sale (Morocco). Annali di Chimica., 90: 695-702.

Fadrowski JJ, Navas-Ancien A, Tellez-Plaza M, Guallar E, Weaver VM, Furth SL. 2010. Blood lead level and kidney function in US adolescents. The third National Health and Nutrition Examination Survey. Arch. Intern. Med., 170: $75-82$.

Farombi EO, Adelowo OA, Ajimoko YR. 2007. Biomarkers of oxydative stress and heavy metal levels as indicators of environmental pollution in African Cat Fish (Clarias gariepinus) from Nigeria Ogun River. International Journal of Environmental Research and Public Health., 4(2): 158-165.

Gallagher CM, Kovach JS, Meliker JR. 2008. Urinary cadmium and osteoporosis in
U.S. Women > or $=50$ years of age: NHANES 1988-1994 and 1999-2004. Environ Health Perspect., 116: 13381343.

Gnandi K, Tobschall HJ. 2002. Heavy metals distribution of soils around mining sites of cadmium rich marine sedimentary phosphorites of Kpogamé and Hahotoé (Southern Togo). Environmental Geology, 41(5): 593-600.

Gnandi K, Tobschall HJ. 2003. Distribution patterns of rare-earth elements and uranium in tertiary sedimentary phosphorites of Hahotoé-Kpogamé, Togo. Journal of African Earth Sciences. 37: $1-10$

Gnandi K, Tchangbedji G, Baba G, Kili K, Abbé KD. 2006a. The impact of phosphate mine tailings on the bioaccumulation of heavy metals in marine fish and crustaceans from the coastal zone of Togo. International Journal of Mine Water and Environment, 25(1): 56-62.

Gnandi K, Tozo K, Amouzouvi K, Baba G, Tchangbedji G, Killi AK, Agbéko K. 2006b. Impact de l'exploitation minière sur la santé humaine : cas de la fluorose dentaire chez les enfants autour de l'usine de traitement des phosphates de Kpémé (Sud-Togo). J. Rech. Sci. Univ. Lomé (Togo), série A, 8(2): 195-205.

Khassouani CE, Soulaymani R, Mauras Y, Allain P. 2000. Blood cadmium concentration in the population of Rabat area. Clinica Chimica Acta., 302: 155160.

Labat L. 2010. Biological matrices preparation for determination of metals. Ann. Toxicol. Anal., 22(2): 81-88.

Mélila M, Poutouli W, Amouzou KS, Tchangbédji G, Thaou M, Doh A. 2012a. Evaluation de l'impact du rejet des déchets phosphatés dans la mer sur la biodiversité marine dans trois localités côtières au Togo à partir des biomarqueurs du stress oxydatif chez Sphyraena barracuda (HECKEL, 1843). Int. J. Biol. Chem. Sci., 6(2): 820-831. 
Mélila M, Poutouli W, Amouzou KS, Tchangbédji G, Tchaou M, Doh A, Goto C. 2012b. Induction du stress oxydatif chez l'homme suite à la bioconcentration des éléments métalliques (cadmium et plomb) par voie trophique à Kpémé (Sud du Togo). Int. J. Biol. Chem. Sci., 6(3): 1263-1270.

Miquel G. 2001. Effet des métaux lourds sur l'environnement et la santé. Rapport d'information $\quad \mathrm{N}^{\circ}$ 261. Office Parlementaire d'évaluation des choix scientifiques et technologiques, p. 365.

NSE/ONUDI (North-South Environment/ Organisation des Nations Unies pour le Développement Industriel). 2007. Réduction des déchets des mines de phosphates dans le GCLME. Projet de démonstration du Togo. Rapport final, $\mathrm{p}$. 144.

OMS (Organisation Mondiale de la Santé). 1980. Exposition aux métaux lourds : limites recommandées d'exposition professionnelle à visée sanitaire. Série de rapports techniques. Genève; $\mathrm{N}^{\circ} 647$. p. 130.

Spector JT, Navas-Ancien A, Fadrowski J, Guallar E, Jaar B, Weaver VM. 2011.
Associations of blood lead with estimated glomerular filtration rate using MDRD, CKD-EPI and serum cystatin C-based equations. Nephrol Dial Transplant., 1093/ndt/gfq/773

Tanouayi G, Gnandi K, Ahoudi H, Ouro-sama K. 2015. La contamination métallique des eaux de surface et des eaux souterraines de la zone minière d'exploitation des phosphates de Hahotoé-Kpogamé (SudTogo) : cas du cadmium, plomb, cuivre et nickel. Larhyss Journal, 21: 35-50.

Tchangbedji G, Kili KA, Savariaul MJ, Lacout JL. 2003. Chemical and structural characterization of natural phosphate of Hahotoé (Togo). Bull. Chim. Soc. Ethiop., 17(2): 139-147.

Testud F. 2005. Pathologie Toxique Professionnelle et Environnementale (3è edn). Eska: Paris ; 672.

Verloo M. 2003. Métaux lourds dans les denrées alimentaires : origine et évolution des teneurs ; Symposium. In Les oligoéléments dans l'alimentation. Belgique; $\mathrm{p}$. 3. 